\title{
GALOIS GROUPS OF MAXIMAL $p$-EXTENSIONS
}

\author{
ROGER WARE
}

\begin{abstract}
Let $p$ be an odd prime and $F$ a field of characteristic different from $p$ containing a primitive $p$ th root of unity. Assume that the Galois group $G$ of the maximal $p$-extension of $F$ has a finite normal series with abelian factor groups. Then the commutator subgroup of $G$ is abelian. Moreover, $G$ has a normal abelian subgroup with pro-cyclic factor group. If, in addition, $F$ contains a primitive $p^{2}$ th root of unity then $G$ has generators $\left\{x, y_{i}\right\}_{i \in I}$ with relations $y_{i} y_{j}=y_{j} y_{i}$ and $x y_{i} x^{-1}=y_{i}^{q+1}$ where $q=0$ or $q=p^{n}$ for some $n \geq 1$. This is used to calculate the cohomology ring of $G$, when $G$ has finite rank. The field $F$ is characterized in terms of the behavior of cyclic algebras (of degree $p$ ) over finite $p$-extensions.
\end{abstract}

In what follows $p$ will be a fixed odd prime and $F$ will be a field of characteristic different from $p$ containing a primitive $p$ th root of unity $\omega$. Let $F(p)$ denote the maximal Galois extension of $F$ whose Galois group $G_{F}(p)=\operatorname{Gal}(F(p) / F)$ is a pro-p-group. An extension $K / F$ is called a $p$ extension if $K \subseteq F(p)$. Note that if $K / F$ is a $p$-extension with $[K: F]=p$ then $K / F$ is Galois and $K=F(\sqrt[p]{d})$, for some $d \in F$.

The cyclic algebra (or "symbol algebra") generated over $F$ by elements $u, v$, subject to relations $u^{p}=a, v^{p}=b$, and $u v=\omega v u$, will be denoted $(a, b)_{F}$ or simply $(a, b)$ when no confusion is possible. Recall that $(a, b)=0$ in the Brauer group, $\operatorname{Br}(F)$, if and only if $b$ is a norm from $F(\sqrt[p]{a})$; in particular, since $p$ is odd, $\left(a^{i}, a^{j}\right)=0$ in $B_{r}(F)$, for all $a \in F=F \backslash\{0\}$ and all $i, j$.

If $G$ is a pro- $p$-group we set $H^{i}(G)=H^{i}(G, \mathbb{Z} / p \mathbb{Z})$. From Merkurjev and Suslin's work [MS], an element of order $p$ in the Brauer group is a product of cyclic algebras so is, in particular, split by $F(p)$. Hence, from Galois cohomology we have a commutative diagram

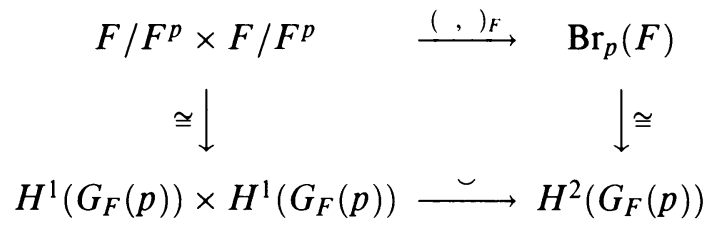

where $\mathrm{Br}_{p} F$ denotes the subgroup of the Brauer group consisting of elements of order $p$. Moreover, if $K=F(\sqrt[p]{d}), G=G_{F}(p), H=G_{K}(p)$, and $\bar{G}=G / H$ then the cohomology sequence $0 \rightarrow H^{1}(\bar{G}) \rightarrow H^{1}(G) \stackrel{\text { res }}{\rightarrow} H^{1}(H)$ corresponds to

Received by the editors February 6, 1990 and, in revised form, July 9, 1990.

1980 Mathematics Subject Classification (1985 Revision). Primary 12F10, $20 \mathrm{E} 18$.

This work was supported in part by NSA research grant no. MDA 904-88-H-2018. 
the sequence $1 \rightarrow\langle a\rangle_{p} \rightarrow F / F^{p} \rightarrow K / K^{p}$ induced by $F \subseteq K$, where $\langle a\rangle_{p}$ is the cyclic subgroup of $F / F^{p}$ generated by $a F^{p}$.

All groups considered here are profinite, homomorphisms are continuous, subgroups are closed, and generating set means topological generating set.

It should be mentioned that when $p=2$ results analogous to those in this paper can be deduced from [JW, Theorems 2.1, 2.3, and Lemma 4.1] and [W, Theorems 4.1, 4.5, and Corollary 4.6]. For other related results the reader is referred to Geyer's paper [G], when $G$ is a "solvable" subgroup of the absolute Galois group of the field of rational numbers, and to Becker's paper [B], in the case that $G$ is the absolute Galois group of a formally real field.

Definition. An element $a$ in $F \backslash F^{p}$ is $p$-rigid if $(a, b)=0$ in $\operatorname{Br}(F)$ implies $b \in a^{i} F^{p}$ for some $i \geq 0$. The field $F$ is called p-rigid if every element in $F \backslash F^{p}$ is $p$-rigid and $F$ is hereditarily p-rigid if every $p$-extension is $p$-rigid. Note that $F$ is hereditarily $p$-rigid iff every finite $p$-extension is $p$-rigid.

Example. If $F$ is a local field with residue field of characteristic not equal to $p$ then $F$ is hereditarily $p$-rigid. Further examples are given in the Corollary and Example following the proof of Theorem 3.

Theorem 1. For the field $F$ the following statements are equivalent:

(a) $F$ is hereditarily p-rigid.

(b) There is an exact sequence $1 \rightarrow \mathbb{Z}_{p}^{1} \rightarrow G_{F}(p) \rightarrow \mathbb{Z}_{p} \rightarrow 1$, for some index set $I$, where $\mathbb{Z}_{p}$ denotes the infinite procyclic p-group.

(c) The commutator subgroup of $G_{F}(p)$ is abelian.

The proof of Theorem 1 requires several lemmas:

Lemma 1. Let $\mu(p)$ be the group of all p-power roots of unity inside $F(p)$. If $\mu(p) \not \subset F$ then $\operatorname{Gal}(F(\mu(p)) / F) \cong \mathbb{Z}_{p}$.

Proof. We fix, inside $F(p)$, a system of primitive roots of unity $\omega_{1}=\omega$, $\omega_{2}, \omega_{3}, \ldots$ chosen so that $\omega_{i}^{p}=\omega_{i-1}$ for all $i$. Then $F(\mu(p))=$ $F\left(\omega_{i} \mid i=1,2, \ldots\right)$. Choose $i \geq 1$ so that $\omega_{i} \in F$ and $\omega_{i+1} \notin F$. Define $x$ on $F(\mu(p))$ by $x\left(\omega_{i+m}\right)=\omega_{i+m}^{p^{i}+1}$. Then restricted to $F\left(\omega_{i+m}\right), x$ has order $p^{m}$ and hence $\operatorname{Gal}(F(\mu(p)) / F)$ is generated by $x$.

For any field $K$ and $a \in \dot{K}$ we set $[a]=a \dot{K}^{p}$. Recall that $\langle a\rangle_{p}$ denotes the cyclic subgroup of $K / K^{p}$ generated by $[a]$.

Lemma 2. Let $K / F$ be a cyclic extension of degree $p$ with generator $\sigma$. For $\beta \in K, K(\sqrt{\beta})$ is Galois over $F$ if and only if $[\sigma \beta]=[\beta]$.

Proof. First assume $K(\sqrt[p]{\beta}) / F$ is a Galois extension. Then $\sqrt[p]{\sigma \beta} \in K(\sqrt[p]{\beta})$ so $[\sigma \beta] \in\langle\beta\rangle_{p}$ (by Kummer theory). If $[\sigma \beta]=[\beta]^{i}$ with $1<i<p$ then in $\dot{K} / \dot{K}^{p},[N(\beta)]=[\beta]^{1+i+i^{2}+\cdots+i^{p-1}}$ where $N: K \rightarrow F$ is the norm. Since $i^{p-1}+i^{p-2}+\cdots+i^{2}+i+1 \equiv 1(\bmod p),[N(\beta)]=[\beta]$ and, because $N(\beta) \in F$, this implies $[\sigma \beta]=[\beta]$.

Conversely, if $[\sigma \beta]=[\beta]$ then $K(\sqrt[p]{\beta})=K(\sqrt[p]{\sigma \beta})$ and $K(\sqrt[p]{\beta}) / F$ is a Galois extension.

Lemma 3. Let $K=F(\sqrt[p]{d}), d \notin F^{p}$, and let $\bar{G}=\operatorname{Gal}(K / F)$. If $\bar{G}$ acts trivially on $K / K^{p}$ then $K / K^{p}=\langle\sqrt[p]{d}\rangle_{p} \times \varepsilon\left(F / F^{p}\right)$, where $\varepsilon$ is the map induced by $F \subseteq K$. 
Proof. By Hochschild-Serre [S, I-15] there is an exact sequence

$$
0 \rightarrow H^{1}(\bar{G}) \rightarrow H^{1}\left(G_{F}(p)\right) \stackrel{\text { res }}{\longrightarrow} H^{1}\left(G_{K}(p)\right)^{\bar{G}} \rightarrow H^{2}(\bar{G})
$$

and since $H^{2}(\bar{G}) \cong \mathbb{Z} / p \mathbb{Z}$, either res is surjective or its image has index $p$ in $H^{1}\left(G_{K}(p)\right)^{\bar{G}}$. Since $\left(\dot{K} / \dot{K}^{p}\right)^{\bar{G}}=\dot{K} / \dot{K}^{p}$, this means the image of $\varepsilon$ has index $p$ or 1 in $\dot{K} / \dot{K}^{p}$. If $[\sqrt[p]{d}] \in \operatorname{Im} \varepsilon$ then $\sqrt[p]{d}=u y^{p}$ with $u \in F, y \in K$. Then $d=$ $N(\sqrt[p]{d})=(u N(y))^{p} \in F^{p}$, a contradiction. Hence $\dot{K} / \dot{K}^{p}=\langle\sqrt[p]{d}\rangle_{p} \times \varepsilon\left(F / F^{p}\right)$.

Recall that for an odd prime $p$ there exist (up to isomorphism) only two nonabelian groups of order $p^{3}$, namely:

Type $\mathbf{E}_{1}$ : Generators $x, y, t$ and relations $x^{p}=y^{p}=t^{p}=1, x y x^{-1} y^{-1}=t$, $x t=t x, y t=t y$.

Type $\mathbf{E}_{2}$ : Generators $x, y$ and relations $x^{p}=y^{p^{2}}=1, x y x^{-1}=y^{p+1}$.

Lemma 4. (1) $F$ is p-rigid if and only if no group of type $\mathrm{E}_{1}$ occurs as a Galois group over $F$.

(2) If $F$ is p-rigid and contains a primitive $p^{2}$ th root of unity then no group of type $\mathrm{E}_{2}$ occurs as a Galois group over $F$; hence, in this case, every Galois extension of degree $p^{3}$ is abelian.

Proof. This is an immediate consequence of [MN, Theorem 14].

Lemma 5. Let $P$ be a p-subgroup of the symmetric group $S_{p^{2}}$. If every subgroup of order $p^{3}$ in $P$ is abelian then $P$ is abelian.

Proof. We may assume $|P|=p^{n}>p^{3}$. The proof proceeds by induction on $n$ so we assume that every subgroup of $P$ of order $p^{n-1}$ is abelian.

We first show that every element in $P$ has order $\leq p$. If not, then $P$ contains an element $y$ of order $p^{2}$. This element must be a $p^{2}$-cycle and hence its centralizer in $P$ is the cyclic subgroup, $\langle y\rangle$, generated by $y$. Since $|P| \geq p^{3}$, the center of $P, Z(P)$, is properly contained in $\langle y\rangle$ and because $P$ is a $p$-group it follows that $Z(P)=\left\langle y^{p}\right\rangle$.

Now let $H$ be a normal subgroup of $P$ of order $p^{n-2}>p$. Then, because $H$ is normal in the $p$-group $P$, the usual argument shows that $|Z(P) \cap H|>1$ and since $|Z(P)|=p$ we conclude that $Z(P) \leq H$. Moreover, $H$ is abelian by the induction assumption.

Case 1. $y \in H$. Then $\langle y\rangle=H$ (because $H$ is abelian and the centralizer of $y$ is $\langle y\rangle)$. Choose $z \in P \backslash H$. Then $z y \neq y z$ so $H\langle z\rangle$ is nonabelian. If $|z|=p$ then

$$
|H\langle z\rangle|=\frac{|H||z|}{|H \cap\langle z\rangle|}=p^{n-2} \cdot p=p^{n-1},
$$

a contradiction. If $|z|=p^{2}$ then by the argument in the second paragraph of this proof (applied there to $y$ of order $p^{2}$ ) we have $Z(P)=\left\langle z^{p}\right\rangle$, hence $z^{p} \in H$. Then $|H\langle z\rangle|=\left(p^{n-2} \cdot p^{2}\right) / p=p^{n-1}$, likewise a contradiction.

Case 2. $y \notin H$. Since $|H|>p$ there exists $h$ in $H$ with $h y \neq y h$. However, $H \cap\langle y\rangle=\left\langle y^{p}\right\rangle$ in this case, yielding $|H\langle y\rangle|=p^{n-1}$, once again contradicting the induction assumption. This completes the proof that every element in $P$ has order $\leq p$. 
Now suppose that $P$ is nonabelian. We assert that in this case $Z(P)$ is the unique normal subgroup of $P$ of order $p^{n-2}$. To see this, let $H$ be a normal subgroup of $P$ with $|H|=p^{n-2}$. If there exists $z$ in $Z(P) \backslash H$ then (since $z$ has order $p) \quad|H\langle z\rangle|=p^{n-1}$ so there exists $x$ in $P \backslash H\langle z\rangle$. Since $H$ is normal in $P, H\langle x\rangle$ is a subgroup of order $p^{n-1}$, hence abelian. If $z \in H\langle x\rangle$ then $z=h x^{i}, 1 \leq i<p$, which forces $x \in H\langle z\rangle$. Hence $z \notin H\langle x\rangle$ so $H\langle x\rangle\langle z\rangle$ is an abelian group of order $p^{n}$, contrary to the assumption that $P$ is nonabelian. Hence $Z(P) \leq H$. On the other hand, if there exists $h$ in $H \backslash Z(P)$ then there exists $x$ in $P \backslash H$ such that $x h \neq h x$ (since $H$ is abelian). But then $H\langle x\rangle$ is a nonabelian group of order $p^{n-1}$. Hence $H=Z(P)$, as asserted.

Still assuming $P$ is nonabelian, let $x, y \in P$ map onto the basis of $P / Z(P) \cong$ $\mathbb{Z} / p \mathbb{Z} \times \mathbb{Z} / p \mathbb{Z}$. Then $P=Z(P)\langle x\rangle\langle y\rangle$ and $x y \neq y x$. However, $x y=z y x$ for some $z \in Z(P)$ which forces $|\langle x, y\rangle| \leq p^{3}$ (as $\left.|x|=|y|=|z|=p\right)$. But then $\langle x, y\rangle$ is abelian by hypothesis.

Lemma 6. Suppose $F$ is hereditarily p-rigid. Let $L$ be a p-extension of $F$ containing a primitive $p^{2}$ th root of unity.

(1) Every p-extension of $L$ of degree $p^{2}$ is a Galois extension.

(2) If $K=L(\sqrt[p]{d}), d \notin L^{p}$, then $K / K^{p}=\langle\sqrt[p]{d}\rangle_{p} \times \varepsilon\left(L / L^{p}\right)$.

(3) If $K$ is a finite p-extension of $L$ then there exist $a_{1}, \ldots, a_{r}$ in $L$ such that

$$
K \subseteq L\left(\sqrt[n_{1}]{a_{1}}, \ldots, \sqrt[p_{r}]{a_{r}}\right)
$$

Proof. (1) Let $M / L$ be a $p$-extension of degree $p^{2}$, let $G=G_{L}(p)$, and $H=$ $G_{M}(p)$. Then $(G: H)=p^{2}$ so there exists a homomorphism $f: G \rightarrow S_{p^{2}}$ with Ker $f \subseteq H$ and whose image $P$ is a $p$-subgroup of $S_{p^{2}}$. Then there exists a Galois $p$-extension $E / L$ containing $M$ such that $\operatorname{Gal}(E / L) \cong P$. By Lemma $4(2)$, every subgroup of $P$ of order $p^{3}$ (if any) is abelian and by Lemma 5, $P$ is abelian. In particular, $H / \operatorname{Ker} f$ is a normal subgroup of $P=G / \operatorname{Ker} f$, whence $H \triangleleft G$.

By (1) and Lemma 2, $\operatorname{Gal}(K / L)$ acts trivially on $K / K^{p}$ so (2) follows from Lemma 3.

To prove (3), we induct on $[K: L]$. Thus we can write $K=M(\sqrt[p]{d})$, with

$$
d \in M \subseteq L\left(\sqrt[p m_{1}]{a_{1}}, \ldots, \sqrt[p_{s}]{a_{s}}\right), \quad a_{i} \in L, m_{i} \geq 0 .
$$

By (2) we may assume $d=u \sqrt[p]{m_{s}} a_{s}$ with $u \in L\left(\sqrt[p]{m_{1}} a_{1}, \ldots, \sqrt[p m_{s}-1]{a_{s}}\right)$ and by the induction assumption

$$
L\left(\sqrt[p m_{1}]{a_{1}}, \ldots, \sqrt[m_{s}-1]{a_{s}}\right)(\sqrt[p]{u}) \subseteq L\left(\sqrt[p^{k_{1}}]{b_{1}}, \ldots, \sqrt[p^{k_{t}}]{b_{t}}\right), \quad b_{i} \in L .
$$

Hence $K \subseteq L\left(\sqrt[p]{m_{1}} a_{1}, \ldots, \sqrt[p m_{s}]{a_{s}}, \sqrt[p]{k_{1}}, \ldots, \sqrt[p]{b_{1}}, b_{t}\right)$.

Lemma 7. Assume $\left|F / F^{p}\right|=p^{2}$. Then either $G_{F}(p)$ is a free pro-p-group (of rank 2) or $G_{F}(p)$ has generators $x, y$ and relation $x y x^{-1}=y^{q+1}$, where $q=0$ or $q=p^{m}, m \geq 1$.

Proof. Choose generators $[a],[b]$ for $F / F^{p}$. If $(a, b)=0$ then $(u, v)=0$ for all $u, v$ in $F$ and by the Merkurjev-Suslin theorem [MS], $H^{2}\left(G_{F}(p)\right)=0$. Hence $G_{F}(p)$ is a free pro-p-group in this case [S, I-37].

If $(a, b) \neq 0$ then the pairing $H^{1}(G) \times H^{1}(G) \rightarrow H^{2}(G), G=G_{F}(p)$, is necessarily nondegenerate so, again using Merkurjev-Suslin, $G$ is a Demushkin 
group of rank 2 and by Demushkin's theorem [D], $G$ has the generators and relation described above.

Remark. Using Merkurjev and Suslin's result it is easy to show that the following statements are equivalent (giving a $p$-analogue of [S, Proposition 5, II-7], when $F$ contains a primitive $p$ th root of unity):

(a) $G_{F}(p)$ is a free pro- $p$-group.

(b) The $p$-primary part, $\operatorname{Br}(F)(p)$, of the Brauer group of $F$ is trivial.

(c) $\operatorname{Br}(K)(p)=0$ for every $p$-extension $K$ of $F$.

(d) For every $p$-extension $K$ of $F$ and every $p$-extension $L$ of $K, N_{L / K}: L$ $\rightarrow K$ is surjective.

(e) For every cyclic extension $K / F$ of degree $p, N_{K / F}: K \rightarrow F$ is surjective.

Proof of Theorem 1. (a) $\Rightarrow(\mathrm{b})$. Let $L=F(\mu(p))$ where, as before, $\mu(p)$ is the group of all $p$-power roots of unity. By Lemma 6, $F(p)=L(p)=$ $L\left(\sqrt[p]{n_{i}} a_{i} \mid i \in I, n_{i} \geq 0\right)$, where $\left\{\left[a_{i}\right]\right\}_{i \in I}$ is an $\mathbb{F}_{p}$-basis for $L / L^{p}$. Since all $p$-power roots of unity lie in $L, \operatorname{Gal}(F(p) / L) \cong \mathbb{Z}_{p}^{I}$ (direct product) and by Lemma $1, \operatorname{Gal}(L / F) \cong \mathbb{Z}_{p}$ or $\{1\}$.

(b) $\Rightarrow(\mathrm{c})$. Given an exact sequence as in (b) the commutator subgroup of $G_{F}(p)$ must be contained in $\mathbb{Z}_{p}^{I}$.

(c) $\Rightarrow(\mathrm{a})$. Suppose $K$ is a $p$-extension of $F$ and $a, b$ are elements of $K$ with $(a, b)=0$. If $[b] \notin\langle a\rangle_{p}$ then $[a],[b]$ are independent over $\mathbb{F}_{p}$. Let $M$ be a maximal $p$-extension of $K$ such that $[a],[b]$ remain linearly independent in $M / M^{p}$. We assert that $M / M^{p}=\langle a\rangle_{p} \times\langle b\rangle_{p}$. Indeed, if $c \in M \backslash M^{p}$ then $L=M(\sqrt[p]{c})$ is a larger extension so there exists $i, j(\operatorname{not} b o t h 0 \bmod p)$ such that $a^{i} b^{j} \in L^{p}$. Kummer theory implies that $[a]^{i}[b]^{j}=[c]^{k}$ in $M / M^{p}$ with $0<k<p$ and hence $[c] \in\langle a\rangle \times\langle b\rangle$. Thus the group $G_{M}(p)$ has rank 2 . Since $(a, b)=0$ the proof of Lemma 7 shows that $G_{M}(p)$ is a free pro- $p$ group. Let $C$ be the commutator subgroup of $G_{M}(p)$. Since the factor group $G_{M}(p) / C$ is a free abelian pro-p-group of rank $2, C$ is a free pro-p-group of infinite rank [S, Proposition 22, Corollary 3, I-33, I-37]. Since $C$ is contained in the commutator subgroup of $G_{F}(p)$ this contradicts (c).

A profinite group $G$ is said to be metabelian if there is an exact sequence $1 \rightarrow A \rightarrow G \rightarrow B \rightarrow 1$ of profinite groups with $A$ and $B$ abelian. It is clear that $G$ is metabelian iff its commutator subgroup is abelian.

Corollary 1. For the group $G=G_{F}(p)$ the following statements are equivalent:

(a) $G$ is not metabelian.

(b) $G$ contains a free pro-p-subgroup of rank 2 .

(c) $G$ contains a free pro-p-subgroup of infinite rank.

Proof. (a) $\Rightarrow(\mathrm{b})$. Let $L=F(\mu(p))$. If $G_{L}(p)$ is abelian then $G$ is metabelian so we can choose $x, y$ in $G_{L}(p)$ with $x y \neq y x$. If the pro- $p$-subgroup generated by $x$ and $y$ is not free then by Lemma 7 it is metabelian and hence by Theorem $1((c) \Rightarrow(a)$ and the proof of $(a) \Rightarrow(b))$ it is abelian.

(b) $\Rightarrow$ (c). As noted in the proof of Theorem 1 (c) $\Rightarrow(a)$, the commutator subgroup of a free pro-p-group of rank 2 is a free pro-p-group of infinite rank.

(b) $\Rightarrow(\mathrm{a})$. Choose $H$ free of rank $2, H \leq G$. Then the commutator subgroup of $H$ is contained in the commutator subgroup of $G$ so the latter cannot be abelian. 
Corollary 2. Assume $G=G_{F}(p)$ has finite rank $r$.

(1) If rank $H \leq r$ for all subgroups $H$ then $G_{F}(p)$ is metabelian.

(2) If $G$ is metabelian and $F$ contains a primitive $p^{2}$ th root of unity then rank $H \leq r$ for all subgroups $H$.

Proof. (1) follows from Corollary 1.

(2) We first show that the rank of $H$ equals $r$, whenever $(G: H)$ is finite. By induction it suffices to assume that $(G: H)=p$. Then the result follows from Theorem 1, (c) $\Rightarrow$ (a), and Lemma 6(2).

For the general case, suppose rank $H>r$. Then there exist $r+1 \mathbb{F}_{p^{-}}$ linearly independent elements $\left[a_{1}\right], \ldots,\left[a_{r+1}\right]$ in $L / L^{p}$, where $L$ is the fixed field of $H$. If $K=F\left(a_{1}, \ldots, a_{r+1}\right)$ then $G_{K}(p)$ has finite index in $G$ and $\operatorname{rank} G_{K}(p) \geq r+1$.

Corollary 3. If $G_{F}(p)$ is metabelian and rank $G_{F}(p)=r$ then

$$
\operatorname{dim}_{\mathbb{F}_{p}} \operatorname{Br}_{p}(F)=\frac{r(r-1)}{2}
$$

Proof. By the Merkurjev-Suslin theorem it suffices to show that if $\left[a_{1}\right], \ldots,\left[a_{t}\right]$ are linearly independent in $F / F^{p}$ then $\left\{\left(a_{i}, a_{j}\right)\right\}_{i<j}$ is a linearly independent subset of $\operatorname{Br}(F)$. If not, among all hereditarily $p$-rigid fields where this fails choose one, $F$, with $t$ minimal. Then there is a relation $\sum_{i<j} n_{i j}\left(a_{i}, a_{j}\right)=0$ with $n_{i j} \in \mathbb{F}_{p}$, not all zero. Let $K=F\left(\sqrt[p]{a_{t}}\right)$. Then $\left[a_{1}\right], \ldots,\left[a_{t-1}\right]$ remain linearly independent in $K / K^{p}$ so by the minimality of $t$, the set $\left\{\left(a_{i}, a_{j}\right)\right\}$, $1 \leq i<j<t$, is linearly independent in $\operatorname{Br}(K)$. This forces $n_{i j}=0$ for $1 \leq i<j<t$ and we are left with $\sum_{i<t} n_{i t}\left(a_{i}, a_{t}\right)=0$ in $\operatorname{Br}(F)$; i.e., $\left(a_{1}^{n_{1 t}} \cdots a_{t-1}^{n_{t-1, t}}, a_{t}\right)=0$. Since $F$ is $p$-rigid this implies $\left[a_{1}^{n_{1 t}} \cdots a_{t-1}^{n_{t-1}, t}\right] \in\left\langle a_{t}\right\rangle_{p}$ contrary to the linear independence of $\left[a_{1}\right], \ldots,\left[a_{t}\right]$.

Remark. In Theorem 4, this corollary will be generalized under the additional assumption that $F$ contains a primitive $p^{2}$ th root of unity.

Theorem 2. Assume $G_{F}(p)$ is a metabelian pro-p-group. If $F$ contains a primitive $p^{2}$ th root of unity then $G_{F}(p)$ has generators $\left\{y_{i}, x\right\}_{i \in I}$ with relations $y_{i} y_{j}=y_{j} y_{i}$ and $x y_{i} x^{-1}=y_{i}^{q+1}$ where $q=0$, if $f$ contains all $p$-power roots of unity, or $q=p^{n}$, where $n$ is the largest integer such that $F$ contains a primitive $p^{n}$ th root of unity.

Proof. If $F$ contains all $p^{m}$ th roots of unity, $m>0$, this follows as in the proof of Theorem 1, (a) $\Rightarrow($ b). Otherwise, by Lemma $6(3), F(p)=$ $F\left(\omega_{n+j}, \sqrt[m_{i}]{a_{i}} \mid j=1,2, \ldots, i \in I, m_{i}>0\right)$ where $\left\{\left[\omega_{n}\right],\left[a_{i}\right]\right\}_{i \in I}$ is an $\mathbb{F}_{p^{-}}$ basis for $F / F^{p}$ and the $\omega_{k}$ are chosen so that $\omega_{1}=\omega$ and $\omega_{k}^{p}=\omega_{k-1}$ (as in the proof of Lemma 1). Thus we can define a set of generators $\left\{y_{i}, x_{i}\right\}_{i \in I}$ for $G_{F}(p)$ as follows:

$$
\begin{gathered}
x\left(\omega_{n+j}\right)=\omega_{n+j}^{q+1}, \quad q=p^{n}, \quad j \geq 1 ; \quad x\left(\sqrt[p m]{a_{i}}\right)=\sqrt[p m]{a_{i}}, \\
y_{i}\left(\sqrt[p m]{a_{i}}\right)=\omega_{m} \sqrt[p]{a_{i}}, \quad y_{i}\left(\sqrt[p]{a_{k}}\right)=\sqrt[p]{a_{k}} \quad \text { if } k \neq i \\
y_{i}\left(\omega_{m}\right)=\omega_{m} \quad \text { for all } m \geq 1
\end{gathered}
$$

It is readily verified that the set $\left\{y_{i}, x\right\}_{i \in I}$ satisfies the given relations. 
Remark. One should be able to remove the assumption on the existence of a $p^{2}$ th root of unity. However the use of Lemma $6(3)$ seems to be crucial for the above proof.

A profinite group $G$ is solvable if there exists a chain of (closed) subgroups $\{1\}=H_{0} \subseteq H_{1} \subseteq \cdots \subseteq H_{n}=G$ with $H_{i} \triangleleft H_{i+1}$ and $H_{i+1} / H_{i}$ abelian.

Theorem 3. The following statements are equivalent:

(a) $G_{F}(p)$ is solvable.

(b) $G_{F}(p)$ is metabelian.

(c) $G_{F}(p)$ does not contain a free, nonabelian subgroup.

Proof. The equivalence of (b) and (c) is contained in Corollary 1 to Theorem 1. It remains to prove $(\mathrm{a}) \Rightarrow(\mathrm{b})$. Assume $G=G_{F}(p)$ is solvable. By induction we may assume $G$ has subgroups $H_{1}, H_{2}$ such that $H_{1} \triangleleft H_{2}, H_{2} \triangleleft G$ and $H_{1}, H_{2} / H_{1}, G / H_{2}$ are abelian. By Theorem 1, the fixed field $F_{2}$ of $H_{2}$ is hereditarily $p$-rigid. Let $L=F(\mu(p))$ and let $L_{2}=F_{2} L$. Then $L_{2}$ is hereditarily $p$-rigid so (because $\left.\mu(p) \subseteq L_{2}\right) \quad G_{L_{2}}(p)$ is abelian. Moreover, there exists an injective homomorphism $G_{L}(p) / G_{L_{2}}(p) \hookrightarrow G_{F}(p) / G_{F_{2}}(p)=G / H_{2}$. Hence $G_{L}(p)$ is metabelian and $L$ is hereditarily $p$-rigid. Since $\mu(p) \subseteq L, G_{L}(p)$ is abelian, whence $G_{F}(p)$ is metabelian.

Let $\Gamma=\mathbb{Z}^{(I)}$ (direct sum) to be totally ordered group obtained by totally ordering the set $I$ and then using the usual lexicographic ordering. Let $F((\Gamma))=$ $\{f: \Gamma \rightarrow F \mid \operatorname{supp}(f)$ is well ordered $\}$ be the (henselian) generalized formal power series field. If $|I|=n$ then $F((\Gamma))$ can be identified with the field of iterated power series $F\left(\left(x_{1}\right)\right) \cdots\left(\left(x_{n}\right)\right)$.

Corollary. $F$ satisfies the conditions of Theorem 1 if and only if $F((\Gamma))$ does.

Proof. Let $K=F((\Gamma))$. From valuation theory there is an exact sequence

$$
1 \rightarrow \mathbb{Z}_{p}^{I} \rightarrow G_{K}(p) \rightarrow G_{F}(p) \rightarrow 1
$$

where $\mathbb{Z}_{p}^{I}$ is identified with $G_{K_{n r}}(p)$, where $K_{n r}$ is the maximal nonramified extension of $K$ inside $K(p)$. Hence $G_{K}(p)$ metabelian implies $G_{F}(p)$ metabelian. On the other hand, if $G_{F}(p)$ is metabelian then $G_{K}(p)$ is solvable and Theorem 3 applies.

Example. Given any pro-p-group $G$ with generators and relations as described in Theorem 2 , there is a field $F$ with $G_{F}(p) \cong G$ :

Let $r$ be a prime with $r \equiv 1(\bmod p)$, let $K=\mathbb{F}_{r}\left(\omega_{n}\right)$ where $\omega_{n}$ is a primitive $p^{n}$ th root of unity (resp., $K=\mathbb{F}_{r}(p)$ ) and let $F=K((\Gamma)), \Gamma=\mathbb{Z}^{(I)}$.

Theorem 4. Assume $G=G_{F}(p)$ is solvable and $F$ contains a primitive $p^{2}$ th root of unity. If $\operatorname{rank} G=n$ then for $k \geq 0, \operatorname{dim}_{\mathbb{F}_{p}} H^{k}(G)=\left(\begin{array}{l}n \\ k\end{array}\right)$ (where $\left(\begin{array}{l}n \\ k\end{array}\right)=0$ if $k>n)$.

Proof. We proceed by induction on $n$. By Theorem 2 there is an abelian subgroup $N$ of rank $n-1$ such that $G / N \cong \mathbb{Z}_{p}$. The Lyndon-HochschildSerre spectral sequence satisfies

$$
E_{2}^{r, s}=H^{r}\left(G / N, H^{s}(N)\right) \Rightarrow H^{r+s}(G) \text {. }
$$

Since $G / N \cong \mathbb{Z}_{p}, E_{2}^{r, s}=0$ for $r \neq 0,1$. Hence as in [R], third quadrant version of Lemma 11.36 , p. 349 , there is an exact sequence

$$
0 \rightarrow E_{2}^{1, k-1} \rightarrow H^{k}(G) \rightarrow E_{2}^{0, k} \rightarrow 0
$$


We assert that $G / N$ acts trivially on $H^{1}(N)$ (and hence on $H^{m}(N)$ for any $m \geq 1)$. The action of $G / N$ on $H^{1}(N)=\operatorname{Hom}(N, \mathbb{Z} / p \mathbb{Z})$ is given by $(\bar{\sigma} \cdot f)(\tau)=f\left(\sigma^{-1} \tau \sigma\right)$, for $\sigma \in G, \tau \in N$. By Theorem 2, $\sigma^{-1} \tau \sigma=\tau^{q+1}$, where either $q=0$ or $q=p^{t}$ for some $t$. Thus $f\left(\sigma^{-1} \tau \sigma\right)=f\left(\tau^{q+1}\right)=$ $(q+1) f(\tau)=f(\tau)$, proving the assertion.

Hence, $E_{2}^{0, k}=H^{0}\left(G / N, H^{k}(N)\right)=H^{k}(N)$ and

$$
E_{2}^{1, k-1}=\operatorname{Hom}\left(G / N, H^{k-1}(N)\right) \cong \operatorname{Hom}\left(\mathbb{Z}_{p}, H^{k-1}(N)\right) \cong H^{k-1}(N) .
$$

Therefore the above sequence becomes

$$
0 \rightarrow H^{k-1}(N) \rightarrow H^{k}(G) \rightarrow H^{k}(N) \rightarrow 0 .
$$

By the induction assumption (and the previous example), $\operatorname{dim}_{\mathbb{F}_{p}} H^{m}(N)=$ $\left(\begin{array}{c}n-1 \\ m\end{array}\right)$. Hence

$$
\operatorname{dim}_{\mathbb{F}_{p}} H^{k}(G)=\left(\begin{array}{l}
n-1 \\
k-1
\end{array}\right)+\left(\begin{array}{c}
n-1 \\
k
\end{array}\right)=\left(\begin{array}{l}
n \\
k
\end{array}\right) .
$$

Corollary. With the assumptions in Theorem 4, the cohomology ring $H^{*}(G)=$ $\bigsqcup_{k \geq 0} H^{k}(G)$ is isomorphic to the exterior algebra over $\mathbb{F}_{p}$ with generators $x_{1}$, $\ldots, x_{n}$.

Remark. If $p=2$ the foregoing argument, together with [JW, Theorem 2.3, and Lemma 4.1], shows that $H^{*}(G)$ is isomorphic to the (commutative) polynomial ring $\mathbb{F}_{2}\left[x_{1}, \ldots, x_{n}\right]$ modulo the ideal generated by $x_{1}^{2}, \ldots, x_{n}^{2}$.

\section{REFERENCES}

[B] E. Becker, Formal-reele Körper mit streng-auflösbarer absoluter Galoisgruppe, Math. Ann. 238 (1978), 203-206.

[D] S. Demushkin, On the maximal p-extension of a local field, Izv. Akad. Nauk SSR Ser. Math. 25 (1961), 329-346.

[G] W.-F. Geyer, Unendliche algebraische Zahlkörper, über denen jede Gleichung auflösbar von beschrankter Stufe ist, J. Number Theory 1 (1969), 346-374.

[JW] B. Jacob and R. Ware, A recursive description of the maximal pro-2 Galois group via Witt rings, Math. Z. 200 (1989), 379-396.

[MN] R. Massy and T. Nguyen-Quang-Do, Plongement d'une extension de degree $p^{2}$ dans une surextension non abelienne de degre' $p^{3}$ : étude locale-globale, J. Reine Angew. Math. 291 (1977), 149-161.

[MS] A. Merkurjev and A. A. Suslin, $K$-cohomology of Severi-Brauer varieties and the norm residue homomorphism, Izv. Akad. Nauk SSSR Ser. Mat. 46 (1982), 1011-1046; English transl., Math. USSR Izv. 21 (1983), no. 2, 307-340.

[R] J. J. Rotman, An introduction to homological algebra, Academic Press, 1979.

[S] J.-P. Serre, Cohomologie Galoisienne, Lecture Notes in Math., vol. 5, Springer-Verlag, 1965.

[W] R. Ware, Quadratic forms and profinite 2-groups, J. Algebra 58 (1979), 227-237.

Department of Mathematics, The Pennsylvania State University, University Park, Pennsylvania 16802

E-mail address: ware@math.psu.edu 